\title{
Eosinophilic sialodochitis: a case report of a rare disease
}

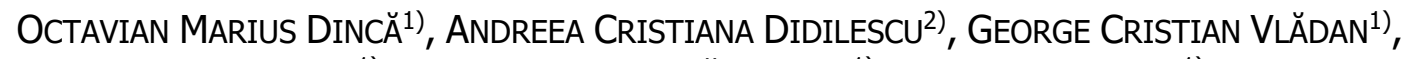 \\ MiHAI BOGDAN BUCUR ${ }^{1)}$, LAVINIA CRISTINA PĂDURARIU ${ }^{1)}$, ALEXANDRU BUCUR ${ }^{1)}$ \\ 1) Department of Oral and Maxillofacial Surgery, Faculty of Dental Medicine, Carol Davila University of Medicine and Pharmacy, \\ Bucharest, Romania \\ 2) Division of Embryology, Faculty of Dental Medicine, Carol Davila University of Medicine and Pharmacy, Bucharest, Romania
}

\begin{abstract}
Eosinophilic sialodochitis (ES) is a rare clinical entity which presents as recurrent major salivary gland swelling and the presence of eosinophilrich mucus plugs or histopathological evidence of eosinophilic infiltration around the larger salivary gland ducts. We present a case of ES not related with underwent a left submaxillectomy because recurrent episodes of submandibular gland swelling associated for the last three years. The laboratory workup demonstrated eosinophils slightly elevated and high levels of immunoglobulin $\mathrm{E}(\mathrm{IgE})$ in peripheral blood. Pathology slides confirmed the final diagnosis of ES, showing a dense periductal eosinophil-rich inflammatory infiltrate and periductal fibrosis. Although the entity is well described in the literature, diagnosis is often difficult due to its clinical presentation being similar to other conditions. Lesions that should be taken into consideration in differential diagnosis are mentioned. This will be the first ES case report in Eastern European literature.
\end{abstract}

Keywords: eosinophilic sialodochitis, salivary gland, eosinophils.

\section{a Introduction}

Eosinophilic sialodochitis (ES) is one of the rarest known conditions that affect the salivary glands. The disease is characterized by recurrent salivary gland swelling associated with mucous plugs or histological evidence of eosinophilic infiltration around the main salivary gland ducts [1-3].

Diagnosis of ES is difficult and may be easily confused with either acute inflammatory disorders or chronic conditions that may subsequently cause salivary gland swelling [4-7].

Criteria for the identification of ES are regularly based on a list created by Baer et al. [4]: recurrent episodes of parotid and/or submandibular glandular swelling; intraductal mucous plugs containing frequent eosinophils; eosinophilia and high levels of immunoglobulin E (IgE) in peripheral blood; associated allergic disease; dilated main salivary ducts; lack of diagnostic criteria for sclerosing disease associated with immunoglobulin G4 (IgG4).

As limitations exist across those criteria, recently Carey et al. [5] propose one algorithm with refined criteria for defining and differentiating ES: $(i)$ intermittent swelling of at least one major salivary gland; (ii) presence of eosinophils in ductal contents; (iii) presence of at least one of the following additional symptoms: itching of the skin overlying the affected gland/pain in the affected gland/ expression of string-like mucous plugs; (iv) exclusion of other causes of salivary gland swelling with eosinophils.

\section{Aim}

Here, we present a rare case of ES, discussing the patient's history, symptoms, diagnosis, and treatment.

\section{Case presentation}

A 49-year-old woman sought treatment at the Prof. Dr. Dan Theodorescu Clinical Hospital of Oral and Maxillofacial Surgery, Bucharest, Romania, in May 2019, because recurring episodes of swelling unrelated to mastication and pain of the left submandibular area during the past three years that does not relieve on common nonsteroidal anti-inflammatory drugs. Episodes occurred 2-3 times per year and are not related with food consumption. Also, the patient stated that during this time she also had two episodes of swelling of the left parotid gland, lasting several hours, which remitted spontaneous. Antispasmodic drugs and massage to compress the salivary gland provide variable symptomatic relief, until the moment when the painful episodes became more severe in frequency and duration.

Physical examination at admission revealed a painless diffuse swelling of the left submandibular area gland that is tender upon palpation. On bimanual palpation left submandibular gland was enlarged and firm miming a chronic sclerosing sialadenitis. Intraoral examination was unremarkable except for palpation of the submandibular duct, which expressed thick mucoid secretions without purulence. Sialoliths were absent. She does not have any palpable cervical nodes. She was not taking any medications and had no history of systemic disorders. Also, she had no past medical history of allergies. The patient quitted smoking five years ago and had no history of alcohol consumption.

The ultrasonography of the submandibular area revealed a heterogeneous echotexture and hypoechoic echogenicity throughout the submandibular gland (Figure 1). Also, we noted dilation of the submandibular ductal system without evidence of sialoliths.

This is an open-access article distributed under the terms of a Creative Commons Attribution-NonCommercial-ShareAlike 4.0 International Public License, which permits unrestricted use, adaptation, distribution and reproduction in any medium, non-commercially, provided the new creations are licensed under identical terms as the original work and the original work is properly cited. 
Routine hematological investigations were within normal limit, except eosinophil count of $3.1 \mathrm{~mL} / \mu \mathrm{L}$ (normal range $0.04-0.45 \mathrm{~mL} / \mu \mathrm{L}$ ). The assays for antinuclear antibodies (ANAs), anti-double-stranded deoxyribonucleic acid (DNA) antibodies, and anti-extractable nuclear antigen antibodies including anti-Sjögren syndrome-related antigen A and antiSjögren syndrome-related antigen $\mathrm{B}$ were all negative; C-reactive protein (CRP), IgG, immunoglobulin A (IgA), immunoglobulin $\mathrm{M}$ (IgM), IgG4, ANA and rheumatoid factor levels were normal, but a higher level of IgE was noted: $260 \mathrm{IU} / \mathrm{mL}$ (normal range $<100 \mathrm{IU} / \mathrm{mL}$ ). We performed allergy tests, including skin prick tests with a standard panel of allergen extracts, patch tests for allergens and haptens, all of which produced negative results.

The left submandibular gland removal was performed. The patient evolved with no postoperative complications.

Tissue specimens were fixed in $10 \%$ neutral buffered formalin for 48 hours followed by paraffin embedding. Five- $\mu \mathrm{m}$-thick serial sections were obtained from each paraffin block. One section was stained with routine Hematoxylin-Eosin (HE) method for histopathological assessment, the other corresponding sections from each case were selected for immunohistochemistry (IHC). IHC was performed to highlight mast cells (MCs) using

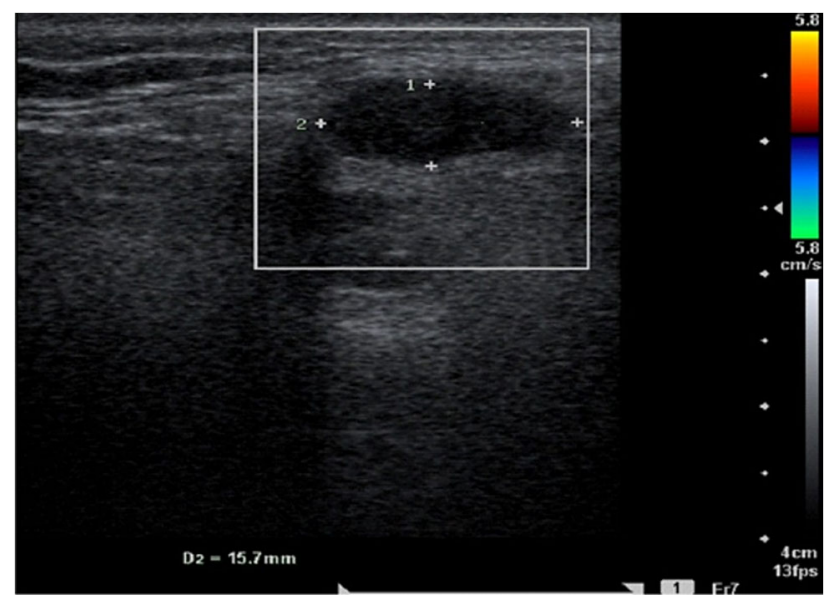

Figure 1 - Ultrasonography shows a slight dilation of left submandibular gland duct.

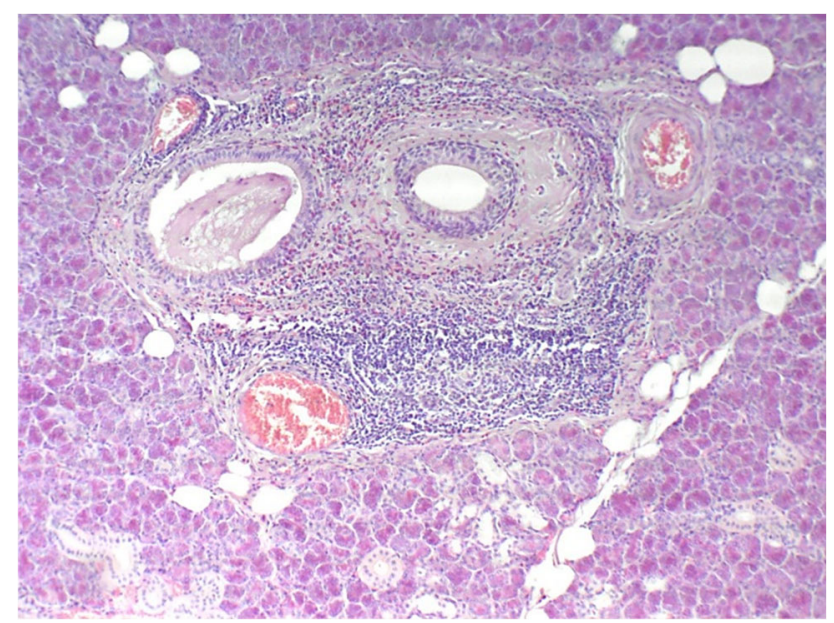

Figure 3 - A HE-stained section in the submandibular gland showing intraductal mucus plug with eosinophils and fibrosis. Adjacent normal acini are present $(\times 100)$. HE: Hematoxylin-Eosin. monoclonal mouse anti-human mast cell tryptase (MCT) antibody, clone 10D11 (Novocastra, Newcastle, UK). Antigen retrieval was achieved by treating sections with a boiling citric acid buffer solution $(0.01 \mathrm{~mol} / \mathrm{L}, \mathrm{pH} 6.0)$ for 10 minutes in a microwave oven. Color was developed in freshly made 3,3'-Diaminobenzidine (DAB). Sections were washed briefly in running tap water and stained lightly with Mayer's Hematoxylin. Negative controls were obtained by omission of the relevant primary antibody. Immunohistochemical procedures were performed in an automatic manner by using Bond-Max Autostainer (Leica Microsystems, Newcastle, UK), which worked with Bond Polymer Refine Detection system.

Gross examination (Figure 2) revealed the left submandibular gland of $4.5 / 3.3 / 1.8 \mathrm{~cm}$, yellow grayish in color, with firm consistency; the lobular contour was preserved; we noted multiple dilations of the intraglandular ducts and marked dilatation of the main submandibular duct, without stones. Histological examination revealed dense eosinophil infiltration around the duct, intraductal mucus plug with eosinophils and fibrin clots and follicular hyperplasia around the ducts (Figures 3-6). Evidence of positive MC infiltration around the large ducts was also found and subsequently confirmed with MCT immunostaining.

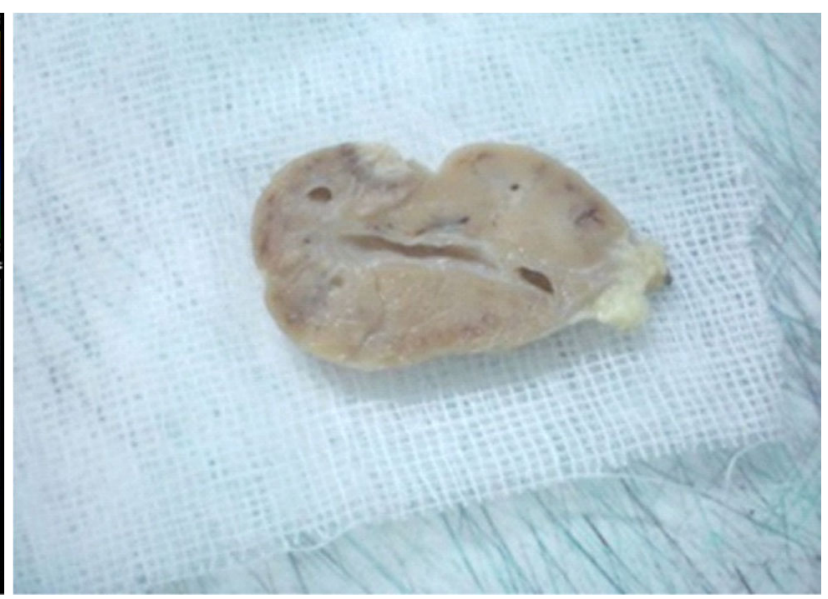

Figure 2 - Cross-section of the left submandibular gland that shows duct ectasia and periductal fibrosis.

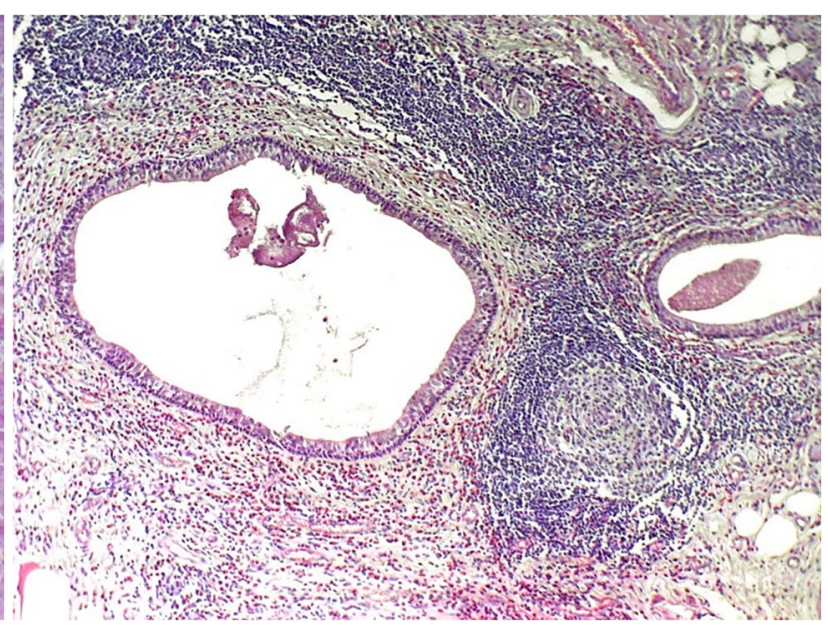

Figure 4 - Microscopic findings of the specimen demonstrating ectasia of interlobular ducts, small amounts of intraluminal mucin, periductal eosinophilic inflammation, fibrosis, marked lymphoplasmacytic inflammation and one large irregular lymphoid follicle with expanded germinal center (HE staining, $\times 100)$. 


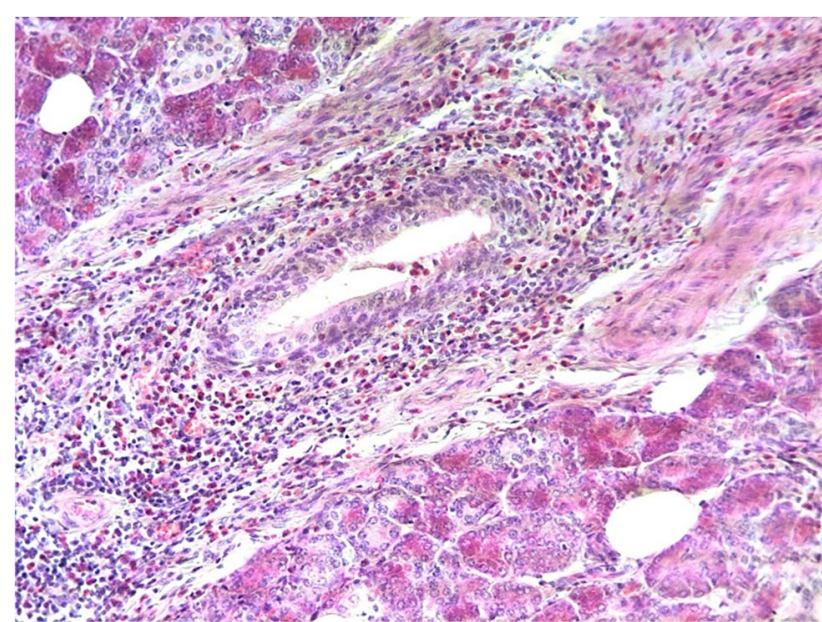

Figure 5 - Microscopic findings of the specimen demonstrating dense eosinophil infiltration and fibrosis around the duct (HE staining, $\times 200)$.

\section{Discussions}

The term "eosinophilic sialodochitis" is considered controversial because the term of sialodochitis is commonly used to describe salivary duct dilatation, associated with sialolithiasis [1]. However, sialodochitis is impossible to separate from sialadenitis clinically and the disease appears initially duct associated. The literature reported that the secretion of a fibrinous material containing numerous eosinophils from the glandular duct orifices was a distinctive finding of ES [1-9]. Few histopathological investigations into ES have been published. However, some studies have reported the presence of eosinophils, and fibrin clots containing detached epithelium in the salivary ducts, while others have described hypertrophy of the salivary duct epithelium, as well as lymphocyte infiltration of the surrounding interstitium $[2,3]$.

In another case report of a patient treated with total parotidectomy, it was noted that fibrin clots continued to be discharged from the opening of the Stensen's duct. This suggests that sialodochitis fibrinosa is an inflammation of the salivary ducts, rather than of the salivary glands [6].

The etiology of ES is not well understood, although the most accepted hypothesis is that it is an allergic process [7-13]. Support for an allergic etiology is based on significant medical history of asthma and chronic rhinoconjunctivitis in some patients and a raised peripheral eosinophil count in affected patients [9]. In our case, we were unable to correlate symptoms with the consumption of food allergens. However, we found numerous eosinophils in the larger glandular ducts, that could be the result of an allergic reaction at the location.

The diagnosis of ES is difficult as many of the symptoms and findings are nonspecific. Thus, it is necessary to rule out the more common causes of salivary gland swelling, such as sialolithiasis, infectious sialadenitis, Sjögren's syndrome or sarcoidosis. Visualizing mucous plugs at the Wharton duct papillae is more common for ES; imaging reveals the absence of calculi and laboratory tests can rule out autoimmune etiologies [2]. Our patient's chief complaint was the recurrent swelling of the left submandibular region over a three-year period. The pressure around the submandibular gland caused the extrusion of the fibrinous material followed by a gush of saliva. We suspected that the material might accompany the secondary

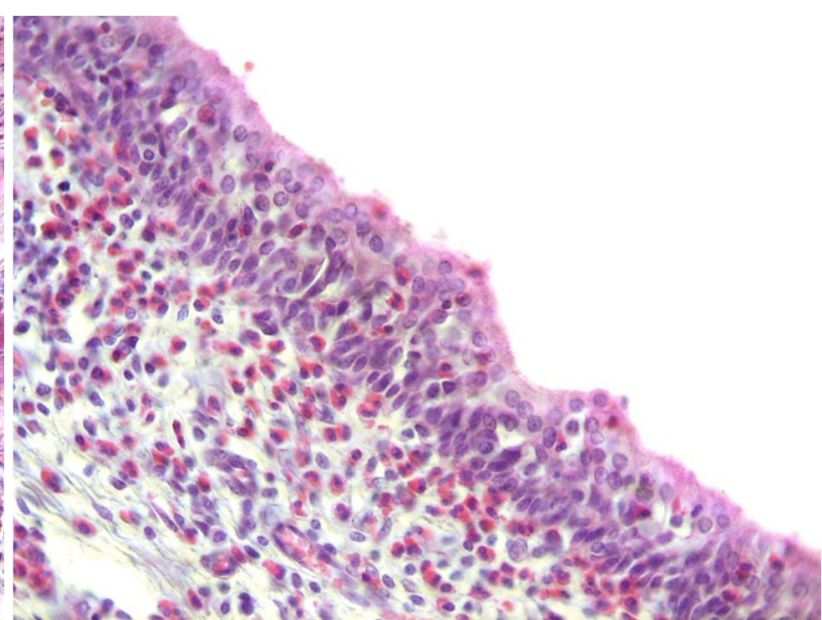

Figure 6 - A section in the submandibular gland revealed marked periductal eosinophilic infiltration and intraepithelial eosinophils (HE staining, $\times 400)$.

bacterial infection of sialolithiasis and performed ultrasonography examination to detect sialoliths. No sialoliths were observed but diffuse swelling of left submandibular gland and dilation of submandibular glandular duct were detected on the ultrasonic images. These findings were consistent with diagnosis of chronic sialodochitis.

Additionally, other diseases that may need to be considered in the differential diagnoses for ES are conditions associated with eosinophils in the salivary gland: Kimura disease, angiolymphoid hyperplasia with eosinophilia and eosinophilic granulomatosis with polyangiitis (EGPA, formerly Churg-Strauss syndrome) $[4,5]$. IgG4-related sialadenitis is characterized by a dense lymphoplasmacytic infiltrate and severe interstitial fibrosis with loss of acini [4]. There was no abnormal level of serum IgG4 elevation in our patients. Kimura's disease presents as subcutaneous painless soft tissue swelling in the head and neck, peripheral blood eosinophils increase, lymphoid follicular hyperplasia and germinal center enlargement, eosinophilic infiltration, and accumulation of eosinophilic micro-abscess [4]. Angiolymphoid hyperplasia with eosinophilia is closely related to Kimura's disease, but histopathology revealed a diffuse inflammatory infiltrate, presence of abundant vascular proliferation with cytoplasmic vacuoles in endothelial cells and eosinophil infiltration $[4,5]$. The salivary glands may rarely be involved in EGPA, a condition characterized by asthma, high levels of eosinophils; major histological findings in this condition are the extravascular granulomas, vasculitis, and the eosinophilic infiltrates [4].

Being a rare condition, no treatment has proven effective [13-19]. Gland resection should be considered in case of recurrence $[6,20]$. With regards to treatment for our patient, she achieved complete remission of her symptoms after excision of the gland. Additionally, the salivary duct was removed at the same time as the gland itself.

\section{a Conclusions}

ES is relatively rare lesion that should be considered in the differential diagnosis of recurrent major salivary gland swelling associated with comorbid atopic disease. According to clinical, ultrasonographic and histological findings associated with increased peripheral eosinophils and total IgE level, the patient was diagnosed with ES. 
Histopathological features included diffuse periductal fibrosis with a lymphoplasmacytic infiltrate rich in eosinophils. These findings were very important for distinguishing ES from Kimura's disease, angiolymphoid hyperplasia with eosinophils and EGPA. The features of these conditions and a differential diagnosis are discussed.

\section{Conflict of interests}

The authors declare that the research was conducted in the absence of any commercial or financial relationships that could be construed as a potential conflict of interests.

\section{Authors' contributions}

O.M.D. and A.B. conceived the original idea and performed data collection. G.C.V. and L.C.P. wrote the main paper. A.C.D. and M.B.B. wrote the Supplementary Information. All authors approved the final version of the manuscript for submission.

\section{Ethics statement}

We obtained the approval of the Ethics Committee of Prof. Dr. Dan Theodorescu Clinical Hospital of Oral and Maxillofacial Surgery, Bucharest, for the publication of this manuscript (No. 6289/30.07.2021).

\section{Consent}

Written informed consent was obtained from patient for the publication of any potentially identifiable images or data included in this article.

\section{Acknowledgments}

The histopathological analysis and the images have been provided by courtesy of Dr. Corina Nastasia, Department of Pathology, Prof. Dr. Dan Theodorescu Clinical Hospital of Oral and Maxillofacial Surgery, Bucharest, Romania, for their availability in diagnosis of the case and the collection of our data.

\section{References}

[1] Pollak N, Templer JW, Esebua M, Diaz-Arias AA, Zitsch RP. Episodic painful parotid swelling caused by sialodochitis with eosinophilic inflammation: a new entity. Otolaryngol Neck Surg, 2009, 140(1):132-133. https://doi.org/10.1016/j.otohns.2008. 10.004 PMID: 19130979

[2] Ray A, Burgin SJ, Spector ME. A rare case of Kussmaul disease (sialodochitis fibrinosa). J Case Rep Med, 2015, 4(1+3):235894. https://doi.org/10.4303/jcrm/235894 PMID: 26246913 PMCID: PMC4523240

[3] Shimada T, Okano H, Hisa Y. A case of severe dilatation of the parotid duct due to fibrinous sialodochitis. Acta Otolaryngol, 2006, 126(10):1112-1114. https://doi.org/10.1080/00016480 600621680 PMID: 16923720

[4] Baer AN, Okuhama A, Eisele DW, Tversky JR, Gniadek TJ. Eosinophilic sialodochitis: redefinition of 'allergic parotitis' and 'sialodochitis fibrinosa'. Oral Dis, 2017, 23(7):840-848. https:// doi.org/10.1111/odi.12595 PMID: 27748012 PMCID: PMC 5393950

[5] Carey B, O’Neill N, Brown J, Escudier M, Hullah E, Beneng K, Odell E, Thomas B, Haque R. Eosinophilic sialodochitis: an emerging atopic condition. Oral Dis, 2021 Mar 4. https://doi. org/10.1111/odi.13821 PMID: 33660371

[6] Hayashi K, Onda T, Ohata H, Takano N, Shibahara T. Case of suspected sialodochitis fibrinosa (Kussmaul's disease). Bull
Tokyo Dent Coll, 2016, 57(2):91-96. https://doi.org/10.2209/ tdcpublication.2015-0028 PMID: 27320298

[7] Zhu WX, Chen Y, Liu DG, Yu GY. Eosinophilic sialodochitis: a type of chronic obstructive sialadenitis related to allergy. Laryngoscope, 2021, 131(3):E800-E806. https://doi.org/10. 1002/lary.28772 PMID: 32621541

[8] Kawamura Y, Ikeda R, Hori T, Sasaki T, Miyabe Y, Fukuchi M, Sakamoto K, Ohta N, Kawase T, Katori Y, Ueki S. Sialodochitis fibrinosa: salivary duct obstruction by eosinophil extracellular traps? Oral Dis, 2020, 26(7):1459-1463. https://doi.org/10. 1111/odi.13434 PMID: 32436360

[9] Flores Robles BJ, Brea Álvarez B, Sanabria Sanchinel AA, Andrus RF, Espinosa Malpartida M, Ramos Giráldez C, Lerma Verdejo A, Merino Argumanez C, Pérez Pimiento JA, Bellas Menéndez C, Villa Alcázar LF, Andréu Sánchez JL, Jiménez Palop M, Godoy Tundidor H, Campos Esteban J, Sanz Sanz J, Barbadillo Mateos C, Isasi Zaragoza CM, Mulero Mendoza JB. Sialodochitis fibrinosa (Kussmaul disease) report of 3 cases and literature review. Medicine (Baltimore), 2016, 95(42): e5132. https://doi.org/10.1097/MD.0000000000005132 PMID: 27759642 PMCID: PMC5079326

[10] Sano T, Miyata J, Sano A, Ono Y, Tanaka Y, Matsukuma S, Ueki S, Kawana A. Eosinophilic sialoadenitis in a patient with severe asthma: a case report. Asia Pac Allergy, 2021, 11(3): e29. https://doi.org/10.5415/apallergy.2021.11.e29 PMID: 34386405 PMCID: PMC8331254

[11] Zhao YN, Zhang LQ, Zhang YQ, Chen Y, Liu DG, Yu GY. Allergy-related sialodochitis: a preliminary cohort study. Laryngoscope, 2021, 131(9):2030-2035. https://doi.org/10. 1002/lary.29508 PMID: 33710620

[12] Salazar P, Celestin J. M104 Sialodochitis fibrinosa: an uncommon and possibly allergic disease of the salivary glands. Ann Allergy Asthma Immunol, 2020, 125(5 Suppl):S67. https://doi.org/10. 1016/j.anai.2020.08.219

[13] Kapoor M, Kelly C. M452 Successful treatment of eosinophilic sialodochitis with dupilumab. Ann Allergy Asthma Immunol, 2020, 125(5 Suppl):S108. https://doi.org/10.1016/j.anai.2020.08.364

[14] Schwarz D, Stuermer KJ, Luers JC. The positive effect of sialendoscopy with irrigation lavage for sialadenitis without sialolithiasis or stenosis. ORL J Otorhinolaryngol Relat Spec, 2018, 80(5-6):271-276. https://doi.org/10.1159/000491492 PMID: 30110688

[15] Pace CG, Hwang KG, Papadaki ME, Troulis MJ. Sialadenitis without sialolithiasis treated by sialendoscopy. J Oral Maxillofac Surg, 2015, 73(9):1748-1752. https://doi.org/10.1016/j.joms. 2015.03.012 PMID: 25863231

[16] Antoniades D, Harrison JD, Epivatianos A, Papanayotou P. Treatment of chronic sialadenitis by intraductal penicillin or saline. J Oral Maxillofac Surg, 2004, 62(4):431-434. https:// doi.org/10.1016/j.joms.2003.07.007 PMID: 15085508

[17] Delagnes EA, Aubin-Pouliot A, Zheng M, Chang JL, Ryan WR. Sialadenitis without sialolithiasis: prospective outcomes after sialendoscopy-assisted salivary duct surgery. Laryngoscope, 2017, 127(5):1073-1079. https://doi.org/10.1002/lary.26308 PMID: 27701754

[18] Marchal F, Becker M, Dulguerov $P$, Lehmann W. Interventional sialendoscopy. Laryngoscope, 2000, 110(2 Pt 1):318-320. https://doi.org/10.1097/00005537-200002010-00026 PMID: 10680937

[19] Capaccio P, Torretta S, Di Pasquale D, Rossi V, Pignataro L. The role of interventional sialendoscopy and intraductal steroid therapy in patients with recurrent sine causa sialadenitis: a prospective cross-sectional study. Clin Otolaryngol, 2017, 42(1): 148-155. https://doi.org/10.1111/coa.12681 PMID: 27200511

[20] Tabaksert AS, Lazarova L, Bentley R, Stafford F. Sialodochitis fibrinosa managed with staged bilateral total parotidectomy. J Laryngol Otol, 2021, 135(2):182-184. https://doi.org/10.1017/ S0022215121000438 PMID: 33593466

\section{Corresponding authors}

George Cristian Vlădan, Lecturer, MD, PhD, Department of Oro-Maxillofacial Surgery, Faculty of Dental Medicine, Carol Davila University of Medicine and Pharmacy, 17-21 Plevnei Avenue, Sector 1, 010221 Bucharest, Romania; Phone +40799-035 042, e-mail: cristi.vladan@umfcd.ro

Andreea Cristiana Didilescu, Professor, DDS, MPhil, MSc, PhD, Division of Embryology, Faculty of Dental Medicine, Carol Davila University of Medicine and Pharmacy, 17-21 Plevnei Avenue, Sector 1, 010221 Bucharest, Romania; Phone +40722-536 798, e-mail: andreea.didilescu@umfcd.ro 